\title{
Analysis of the Behavior of Electric Vehicle Charging Stations with Renewable Generations
}

\author{
Woongsup Lee*, Lin Xiang*, Robert Schober*†, and Vincent W.S. Wong ${ }^{\dagger}$ \\ ${ }^{*}$ Institute for Digital Communications, Friedrich-Alexander University Erlangen-Nurnberg, Erlangen, Germany \\ ${ }^{\dagger}$ Department of Electrical and Computer Engineering, University of British Columbia, Vancouver, Canada
}

\begin{abstract}
In this paper, we study the competitive interactions between electric vehicle charging stations (EVCSs) with renewable electricity generation facilities (REGFs). As electric vehicles (EVs) become more popular, there will be a competition between neighboring EVCSs to attract more EVs. Therefore, an EVCS is likely to set its electricity price by taking into account the competition with neighboring EVCSs, such that its revenue is maximized. We model the competitive interactions between EVCSs by using game theory. In this paper, we show that the game played by EVCSs is a supermodular game and there exists a unique pure Nash equilibrium for best response algorithms with arbitrary initial policy. Simulation results confirm the convergence of the game between EVCSs. The results also verify that it is beneficial for both EVs and EVCSs to have REGFs and all EVCSs will have REGFs in the long run.
\end{abstract}

\section{INTRODUCTION}

Smart grid is the next generation power grid and expected to be distributed, reliable, and more efficient than the current power grid [1]. It is envisioned that the generation of electricity from renewable sources will be more common and will constitute a large portion of the total electricity generation [1]. Therefore, renewable power generation will play a significant role in smart grid and analyzing its effects is necessary.

Another key characteristic of the smart grid is the extensive use of electric vehicles (EVs) [1]. Although the capacity of the battery of a single EV is not large (e.g., $41.8 \mathrm{kWh}$ for Toyota RAV 4), the number of EVs will be large such that the sum capacity of the EV batteries will be substantial (e.g., $106 \mathrm{GWh}$ if $1 \%$ of all vehicles in the U.S. are EVs). Therefore, EVs are considered as one of the major consumers of electricity in smart grid [2]. These numbers are expected to grow considerably over the next few years. As a natural consequence, analyzing the effect of EV charging on smart grid and designing an optimal charging strategy for EVs are crucial [2]- [8].

The most straightforward strategy for charging EVs is to charge EVs when the price of electricity is low, e.g., at night time [3]. In [2], the fluctuation of the power level of the grid was controlled by managing the charging of EVs. The effect of power losses on the transmission line and a departure penalty were taken into account in [5]. Moreover, EV charging was analyzed and optimized by using state-of-the-art mathematical techniques. In [4], the behavior of EVs which try to be charged at minimum cost was analyzed using a mean-field game. Particle swarm optimization was applied in [6] to find the optimal EV charging schedule. In [7], a distributed congestion control for Internet traffic was modified to control EV charging in a distributed manner. Furthermore, consumer preferences for EV charging were investigated in [8].

Most of the existing works on EV charging assumed that EVs are charged only at home. However, considering that conventional internal combustion engine vehicles refuel at gas stations, EVs might also be charged at other facilities which provide charging service. Hereafter, we refer to facilities which provide charging service to EVs as electric vehicle charging stations (EVCSs) [10]. The charging of EVs at EVCSs is viable for the following two reasons: 1) An EVCS can buy electricity from the power grid at a cheaper rate compared to the rate that an EV owner has to pay to charge at home ${ }^{1}$ such that EV charging at the EVCS is cheaper that the charging at home. 2) An EV might run out of electricity while traveling and immediate charging becomes unavoidable $^{2}$. Since charging an EV is more time consuming than refueling a conventional vehicle, the EVCSs can be located at public parking lots and shopping centers where the EVs are parked for a long time [10]. There are some previous works that consider the operation of EVCS. In [12], the optimal deployment of EVCSs was investigated, and in [13], the effect of local energy storage at the EVCSs was investigated.

Currently, the majority of the EVCSs provide electricity at a very cheap rate to stimulate the EV market [10]. However, after the establishment of a stable EV market, the electricity price will be rationalized and EVCSs will generate revenue from selling electricity to EVs. Therefore, when multiple EVCSs with different ownerships coexist in the same area, the competition between the EVCSs to attract EVs is inevitable. An EVCS can attract more EVs by providing electricity at a cheaper price compared to its neighboring competitors. As a consequence, the price of electricity can be different for different EVCSs which is in analogy to the heterogeneity of the gas price at different gas stations. Although the competition between EVCSs is obvious, it has not been considered in previous works on smart grid. There are some previous works which consider the competition between gas stations, e.g., [14]. However, the analysis for gas stations is not directly

\footnotetext{
${ }^{1}$ For example, in the U.S. [11], the price of electricity for the residential sector is $30 \%$ higher than that for the commercial sector. Moreover, many countries adopt tiered electricity rates and charging an EV at home can greatly increase the electricity bill of the EV owner [9].

${ }^{2}$ The distance which an EV can travel on a fully charged battery is generally less than the distance that an internal combustion engine vehicle can travel on a full gas tank.
} 
applicable to EVCSs, because unlike gas stations, EVCSs can generate electricity without incurring additional generation cost by using their own renewable electricity generation facilities (REGFs) such as photovoltaic (PV) generators [10]. In other words, unlike gas stations, EVCSs can generate revenue from generating electricity as well as earning commission for selling electricity to EVs. The use of REGFs by EVCSs is viable because EVCSs can be located at public parking lots and shopping centers which can accommodate REGFs with high generation capacity such as large-size PV generators [22]. Such PV generators may supply more than $500 \mathrm{~kW}$ of power [22] providing sufficient electricity to charge tens of EVs at a time. We note that some EVCSs with REGFs are already in operation [10].

Herein, we analyze the competition between EVCSs with REGFs. The contributions of this paper are as follows:

1) We model the competition between EVCSs with REGFs by using game theory. We show that the resulting game is a supermodular game and there is a unique pure Nash equilibrium for best response algorithms with arbitrary initial values.

2) We evaluate the effect of renewable power generation on the operation of EVCSs and EVs. We show that by adopting REGFs at the EVCSs, the electricity price can be reduced such that EVs can be charged at a cheaper rate while the revenue of the EVCSs improves compared to when the EVCSs sell all electricity generated by the REGFs to the power grid. We also show that the revenue of EVCSs, which are not equipped with REGFs, drops significantly due to the price competition with EVCSs with REGFs and eventually, all EVCSs are likely to employ REGFs.

The rest of this paper is organized as follows. We present the system model in Section II. In Section III, the game model for competing EVCSs is formulated and the supermodularity of the game is proved. The existence of and convergence to a unique Nash equilibrium is also shown in Section III. Simulation results are provided in Section IV. The paper is concluded in Section V.

\section{SYSTEM MODEL}

In this section, we present an analytical description of the operation of EVs and EVCSs. Our system model is depicted in Fig. 1.

\section{A. Electric Vehicles}

We assume that the number of EVs which want to charge their batteries are uniformly distributed in the considered area. An EV selects an EVCS for charging based on the electricity price of the EVCS and its distance to the EVCS. Furthermore, Huff has shown that the preference of customers to a facility is significantly affected by the distance to the facility [15]. Let the penalty that distance has on the selection of an EVCS be modeled as $g\left(d_{i, j}\right)$, where $d_{i, j}$ is the distance between EVCS $i$ and $\operatorname{EV} j$. Then, EV $j$ will choose $\operatorname{EVCS} i^{*}$ if it satisfies

$$
i^{*}=\underset{i \in I}{\operatorname{argmin}} g\left(d_{i, j}\right)+p_{r p}^{i}
$$

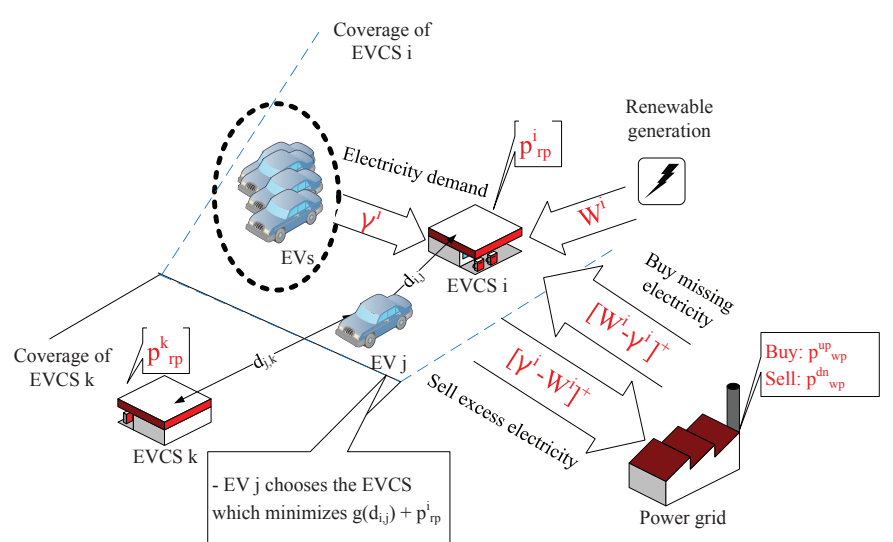

Fig. 1. System model of EVs and EVCSs.

where $I$ is the set of all EVCSs and $p_{r p}^{i}$ is the electricity price of EVCS $i$. Moreover, we assume that the EV reserves the charging service from the EVCS prior to its arrival because the electricity price might change on the way to the EVCS.

\section{B. Operation of Electric Vehicle Charging Stations}

We assume that there are multiple EVCSs under different ownership and the EVCSs are capable of generating electricity from REGFs without incurring additional generation cost. We also assume that an EVCS has to maintain its electricity price fixed for at least $\Delta t$ and the amount of electricity that EVCS $i$ can generate within $\Delta t$ from its own REGFs is $W^{i}$. $W^{i}$ will be significantly affected by the surrounding environment. For example, at night time, we always have $W^{i}=0$ when a PV generator is used. Furthermore, we assume that the EVCSs do not have local energy storage, because storage is expensive [13], and that the number of charging plugs at each EVCS are sufficient to allow charging all of EVs which visit the EVCS.

The total average amount of electricity demand that is incurred to EVCS $i$ within $\Delta t$ is denoted by $\gamma^{i}$, and $N^{i}$ is the average number of EVs which choose EVCS $i$ based on (1). Moreover, let $C_{\text {avg }}$ be the average amount of electricity required for charing one EV. Furthermore, let $B_{a v g}$ and $S_{a v g}^{s o c}$ be the average battery capacity of EVs and the average stateof-charge (SoC) of an EV which visits an EVCS, and the EV is fully charged at the EVCS. Then, $C_{a v g}$ and $\gamma^{i}$ can be modeled as

$$
\begin{aligned}
C_{a v g} & =B_{\text {avg }}\left(1-S_{\text {avg }}^{\text {soc }}\right) \\
\gamma^{i} & =C_{\text {avg }} N^{i} .
\end{aligned}
$$

Each EVCS has two options for selling the excess electricity generated by its REGF. First, the EVCS can sell its excess electricity to the power grid at price $p_{w p}^{u p}$. Second, it can sell the excess electricity at price $p_{r p}^{i}$ to EVs by charging them, see Fig. 1. The value of $p_{w p}^{u p}$ is determined by the power grid and it is likely identical to the wholesale electricity price. However, EVCS $i$ can freely set $p_{r p}^{i}$ to maximize its revenue and $p_{r p}^{i}$ will generally be greater than $p_{w p}^{u p}$, because otherwise there is no incentive for the EVCS to sell its excess electricity to EVs.

When $\gamma^{i}>W^{i}$, the amount of electricity from the REGF is not sufficient to satisfy the electricity demand. Then, EVCS $i$ buys extra electricity from the power grid at price $p_{w p}^{d n}$ and 
correspondingly, the EVCS pays $p_{w p}^{d n}\left(\gamma^{i}-W^{i}\right)$ to the power grid. Otherwise, when $\gamma^{i} \leq W^{i}$, the amount of electricity from the REGF of EVCS $i$ is sufficient to satisfy the electricity demand of the EVs visiting EVCS $i$. In this case, the excess electricity, $W^{i}-\gamma^{i}$, is sold to the power grid at price $p_{w p}^{u p}$. The revenue that EVCS $i$ can get from the power grid by selling the excess electricity is $p_{w p}^{u p}\left(W^{i}-\gamma^{i}\right)$. In general, $p_{w p}^{u p} \leq p_{w p}^{d n}$, because $p_{w p}^{u p}$ is the wholesale price while $p_{w p}^{d n}$ is the retail price.

\section{Coverage of Electric Vehicle Charging Stations}

Herein, we define the coverage of EVCS $i$ as the region in which the EVs will visit EVCS $i$ for charging because EVCS $i$ satisfies the selection criterion of the EV. The coverage of EVCS $i$ can be found by solving (1) for the considered area. Let $N_{a}$ be the total number of EVs in the area. Also, let $S^{\text {area }}$ be the total area that EVCSs can be located in. In this paper, we assume that $N^{i}$ is proportional to the size of the coverage area of EVCS $i$, which we refer to as $S^{i}$, because the EVs are uniformly distributed. Then, $\gamma^{i}$ can be calculated as

$$
\gamma^{i}=C_{a v g} N_{a} \frac{S^{i}}{S^{a r e a}}
$$

The shape of the coverage area of the EVCSs depends on the selection criterion in (1). For general functions $g\left(d_{i, j}\right)$, the boundaries of the coverage areas are not straight lines and will have curvature. Therefore, the coverage areas of the EVCSs are not polygons which makes the analysis complicated. However, it is shown in the following lemma that if $g\left(d_{i, j}\right)$ in (1) has a unary quadratic form such that $g\left(d_{i, j}\right)=\zeta_{1} d_{i, j}^{2}$, where $\zeta_{1}$ is a constant which characterizes the severity of the penalty of distance, then the boundaries of the coverage areas are straight lines and the coverage area of each EVCS is a polygon. The proof of Lemma 1 is omitted here due to page limitations but the complete proof can be found in the longer version of our paper in [23].

Lemma 1. The boundaries of the coverage areas of the EVCSs are straight lines, if $g\left(d_{i, j}\right)$ is a unary quadratic function, i.e., $g\left(d_{i, j}\right)=\zeta_{1} d_{i, j}^{2}$.

\section{A. Game Model}

\section{Price Adjustment Game}

In our system model, the EVCSs will not cooperate because they belong to different owners. As a consequence, each EVCS will set its electricity price independently and greedily by taking into account the electricity price of neighboring EVCSs such that its own revenue is maximized. Herein, we formulate the competitive interactions between EVCSs as a non-cooperative game where each EVCS makes decisions independently as follows.

Price Adjustment Game Between EVCSs:

- Players: EVCSs.

- Strategy: The strategy of the players is the adjustment of the electricity price for charging EVs. In other words, the strategy of EVCS $i$ is setting $p_{r p}^{i}$ where $p_{r p}^{i} \in \mathbb{R}^{+}$. We will use $\mathbf{p}_{r p}^{-i}$ to denote the vector of the electricity prices of all EVCSs except EVCS $i$.
- Payoff: The payoff of EVCS $i, \pi^{i}\left(p_{r p}^{i}, \mathbf{p}_{r p}^{-i}\right)$, is the monetary revenue that EVCS $i$ obtains.

In our system model, EVCS $i$ can generate revenue by charging EVs and the amount of revenue is $p_{r p}^{i} \gamma^{i}$. EVCS $i$ can also obtain revenue from the power grid by selling the excess electricity from the REGF, which remains after satisfying the electricity demand of the EVs. In this case, the amount of revenue is $p_{w p}^{u p} \times\left[W^{i}-\gamma^{i}\right]^{+}$. Finally, EVCS $i$ has to buy additional electricity from the power grid if the amount of electricity from its own REGF, i.e., $W^{i}$, is not sufficient to satisfy the electricity demand of the EVs. In this case, the EVCS should pay for the electricity which is bought from the power grid and the payment is $p_{w p}^{d n} \times\left[\gamma^{i}-W^{i}\right]^{+}$. Therefore, the total revenue of EVCS $i, \pi^{i}\left(p_{r p}^{i}, \mathbf{p}_{r p}^{-i}\right)$, is obtained as

$$
\begin{aligned}
\pi^{i}\left(p_{r p}^{i}, \mathbf{p}_{r p}^{-i}\right)= & p_{r p}^{i} \gamma^{i}\left(p_{r p}^{i}, \mathbf{p}_{r p}^{-i}\right) \\
& +p_{w p}^{u p} \times\left[W^{i}-\gamma^{i}\left(p_{r p}^{i}, \mathbf{p}_{r p}^{-i}\right)\right]^{+} \\
& -p_{w p}^{d n} \times\left[\gamma^{i}\left(p_{r p}^{i}, \mathbf{p}_{r p}^{-i}\right)-W^{i}\right]^{+} \\
= & p_{r p}^{i} \gamma^{i}\left(p_{r p}^{i}, \mathbf{p}_{r p}^{-i}\right)+h\left(\gamma^{i}\left(p_{r p}^{i}, \mathbf{p}_{r p}^{-i}\right)-W^{i}\right)
\end{aligned}
$$

where $h(x)=p_{w p}^{u p} \times[-x]^{+}-p_{w p}^{d n} \times[x]^{+}$. Herein, $\gamma^{i}$ is a function of $p_{r p}^{i}$ and $\mathbf{p}_{r p}^{-i}$, because the demand for electricity at EVCS $i$ depends not only on the electricity price of EVCS $i$ but also on the prices of the other EVCSs, i.e., $\mathbf{p}_{r p}^{-i}$.

$h(x)$ is a piece-wise linear and concave function because $p_{w p}^{u p} \leq p_{w p}^{d n}$. Although $h(x)$ is continuous in the entire range of $x$, it is not differentiable at $x=0$. For the following analysis, we have to differentiate $\pi^{i}\left(p_{r p}^{i}, \mathbf{p}_{r p}^{-i}\right)$ with respect to $p_{r p}^{i}$ to prove the super-modularity of the considered game and this non-differentiable point is problematic. To overcome this problem, we approximate $h(x)$ by smoothing the function around $x=0$, such that the approximated function is differentiable in the entire range of $x$. The approximation of $h(x)$, which we will refer to as $\bar{h}(x)$, is given by

$$
\bar{h}(x)= \begin{cases}\frac{p_{w p}^{d n}-p_{w p}^{u p}}{4 \delta_{a}} \times\left(x+\delta_{a}\right)^{2}+p_{w p}^{u p} x, & \text { for }|x| \leq \delta_{a} \\ h(x), & \text { otherwise. }\end{cases}
$$

We note that for $\delta_{a} \rightarrow 0$, we get $\bar{h}(x) \rightarrow h(x)$. Therefore, $h(x)$ can be approximated by $\bar{h}(x)$ by using a sufficiently small $\delta_{a} \cdot \bar{h}(x)$ is differentiable and concave because $\frac{\partial^{2} \bar{h}(x)}{\partial x^{2}} \leq$ 0 . In the following, we will use $\bar{h}(x)$ instead of $h(x)$.

\section{B. Convergence of the Game}

In the following, we show that the price adjustment game of the EVCSs is a supermodular game. First, let $S$ be the set of strategies that players can choose. Then, the supermodularity of payoff function $\pi^{i}(\mathbf{x})$ is defined as follows [16]:

Definition 1. Payoff function $\pi^{i}(\mathbf{x})$ is supermodular if and only if the following inequality holds:

$$
\pi^{i}(\mathbf{x} \wedge \mathbf{y})+\pi^{i}(\mathbf{x} \vee \mathbf{y}) \geq \pi^{i}(\mathbf{x})+\pi^{i}(\mathbf{y}), \quad \forall \mathbf{x}, \mathbf{y} \in S
$$

where $\mathbf{x} \wedge \mathbf{y}$ and $\mathbf{x} \vee \mathbf{y}$ are the component-wise maximum and minimum of vectors $\mathbf{x}$ and $\mathbf{y}$, respectively.

If $\pi^{i}(\mathbf{x})$ is twice differentiable, then $\pi^{i}(\mathbf{x})$ is supermodular if the following inequality holds [16] 


$$
\frac{\partial^{2} \pi^{i}(\mathbf{x})}{\partial x_{i} \partial x_{j}} \geq 0, \quad \forall \mathbf{x} \in S, j \neq i
$$

Let $S_{i}$ be the set of strategies for player $i$. Also let $s_{i}$ and $\mathbf{S}_{-i}$ be the strategy of player $i$ and the strategies of all players except player $i$, respectively. Then, a supermodular game is defined as follows [17]:

Definition 2. A game is a supermodular game, if 1) the set of strategies of player $i, S_{i}$, is a compact subset of $\left.\mathbb{R}, 2\right) \pi^{i}$ is continuous in $s_{i}$ and $\mathbf{s}_{-i}$, and 3) $\pi^{i}$ is supermodular.

Supermodular games have some nice properties. Especially, pure Nash equilibria exist in supermodular games and the convergence of best response algorithms to the Nash equilibria is guaranteed [16]. Now, we will show that the price adjustment game of the EVCSs is a supermodular game when $g\left(d_{i, j}\right)$ is a unary quadratic function. The supermodularity of the game is formally stated in Theorem 1. The proof of Theorem 1 is omitted here due to page limitations but the complete proof can be found in the longer version of our paper in [23].

Theorem 1. The electricity price adjustment game of the EVCSS is a supermodular game if $g\left(d_{i, j}\right)$, the effect that distance has on the selection of an EVCS, is a unary quadratic function, i.e., $g\left(d_{i, j}\right)=\zeta_{1} d_{i, j}^{2}$.

In Theorem 1 , we assume that $g\left(d_{i, j}\right)$ is a unary quadratic function. Considering Huff's work on the attractiveness of competing facilities which have different distances to customers [15], this assumption seems reasonable. Huff has shown that if two facilities are competing with each other to attract users, the probability that a user goes to one facility is inversely proportional to the square of the distance to the facility. From this, we conjecture that the preference of customers for one facility is inversely proportional to the square of the distance and the penalty of distance in EVCS selection is proportional to the square of the distance.

Based on Theorem 1, a pure Nash equilibrium can be achieved with best response algorithms in the price adjustment game. However, it is not guaranteed that the Nash equilibrium is unique and that the equilibrium can be achieved when the initial strategies are arbitrarily chosen and the strategy of each player is updated in an arbitrary time instant. Therefore, in the following, we will show that a unique pure Nash equilibrium can be achieved by any best response algorithm with any initial policy. To prove the uniqueness and convergence of the game, we need the following two lemmas.

Lemma 2. If $g\left(d_{i, j}\right)$ is a unary quadratic function, i.e., $g\left(d_{i, j}\right)=\zeta_{1} d_{i, j}^{2}, \pi^{i}\left(p_{r p}^{i}, \boldsymbol{p}_{r p}^{-i}\right)$ is a strictly concave function of $p_{r p}^{i}$, i.e., $\frac{\partial^{2} \pi^{i}\left(p_{r p}^{i}, \boldsymbol{p}_{r p}^{-i}\right)}{\left(\partial p_{r p}^{i}\right)^{2}}<0$.

Lemma 3. Let $B r_{i}\left(\boldsymbol{p}_{r p}^{-i}\right)$ be the best response of EVCS $i$ when the electricity price of the other EVCSs is $\boldsymbol{p}_{r p}^{-i}$. Then, add $\eta$ to all components of $\boldsymbol{p}_{r p}^{-i}$ and let this modified electricity price be $\boldsymbol{p}_{r p}^{-i^{\eta}}$, i.e., $p_{r p}^{k^{\eta}}=p_{r p}^{k}+\eta$ for $k \neq i$. Then, if $g\left(d_{i, j}\right)=\zeta_{1} d_{i, j}^{2}$ and $\eta>0, B r_{i}\left(\boldsymbol{p}_{r p}^{-i}\right)+\eta>B r_{i}\left(\boldsymbol{p}_{r p}^{-i^{\eta}}\right)$. In other words, even if all other EVCSs increase their prices by $\eta$, the best response of EVCS $i$ is to increase its price by less than $\eta$.
The proofs of Lemma 2 and Lemma 3 have been omitted here due to the page limitations. The complete proofs can be found in the longer version of our paper in [23]. Based on these two Lemmas and the Theorems 1 and 2 in [16], which consider the uniqueness and convergence of supermodular games, we obtain the following theorem.

Theorem 2. The electricity price adjustment game for EVCSs has a unique pure Nash equilibrium and the equilibrium can be achieved by any best response algorithm with any initial policy, if $g\left(d_{i, j}\right)=\zeta_{1} d_{i, j}^{2}$.

The proof of Theorem 2 has been omitted here due to the page limitations. The complete proof can be found in the longer version of our paper in [23].

\section{PERformance EVAluation}

In this section, we verify the convergence of the price adjustment game. We also evaluate the electricity price and revenue of the EVCSs and illustrate the effect of renewable power generation.

\section{A. Simulation Environment}

Our performance evaluation is conducted based on a realistic simulation environment. In particular, we assume that the electricity price of EVCSs can change every 30 minutes, i.e., $\Delta t=30 \mathrm{~min}$, because it takes about 30 minutes to fully charge the battery of an EV if fast charging is used [10], [13]. We also assume that four EVCSs are located randomly in a $4 \mathrm{~km}$ by $4 \mathrm{~km}$ square area in Philadelphia, PA, USA, based on the actual density of gas stations in this region and an EV market penetration of $13 \%{ }^{3}$. We do not limit the number of EVs that can be charged concurrently in one EVCS. Moreover, we assume that EVs charge their batteries once per day and the average number of EVs that visit the four EVCSs in 30 minutes, $N_{a}=80$ by taking into account the number of registered vehicles in Philadelphia, which is 689,132 [19]. We also assume that $g\left(d_{i, j}\right)=\frac{1}{2} d_{i, j}^{2}$.

Furthermore, we assume that the battery capacity of each $\mathrm{EV}$ is $41.8 \mathrm{kWh}$ which corresponds to the battery capacity of a Toyota RAV 4. Moreover, we assume that the average SoC of EVs which visit an EVCS is $10 \%$ [20] such that $B_{a v g}=37.62$ $\mathrm{kWh}$. For electricity prices, we use the average wholesale and retail electricity prices in Philadelphia in 2011. The average wholesale electricity price is 5.426 cents/kWh and the average retail electricity prices are 13.26 cents $/ \mathrm{kWh}, 10.03$ cents $/ \mathrm{kWh}$, and 7.73 cents/kWh for the residential, commercial, and industrial sectors, respectively [11]. Therefore, we assume that $p_{w p}^{d n}$ is 10.03 cents/kWh and $p_{w p}^{u p}$ is 5.426 cents $/ \mathrm{kWh}$.

For REGF, PV generation is assumed. In Philadelphia, 3.95 $\mathrm{kWh}$ of electricity can be generated per square meter PV panel [21] in one day on average. Herein, we assume that a 5329 $\mathrm{m}^{2}$ commercial PV generation facility is used [22]. We also assume that a REFG only generates electricity during day time which is assumed to last for 12 hours. Thus, a REGF

\footnotetext{
${ }^{3}$ There are 719 gas stations in Philadelphia and the density of gas stations is $1.95 / \mathrm{km}^{2}$. Therefore, the density of EVCSs will be $0.25 / \mathrm{km}^{2}$, if the EV market penetration is $13 \%$.
} 


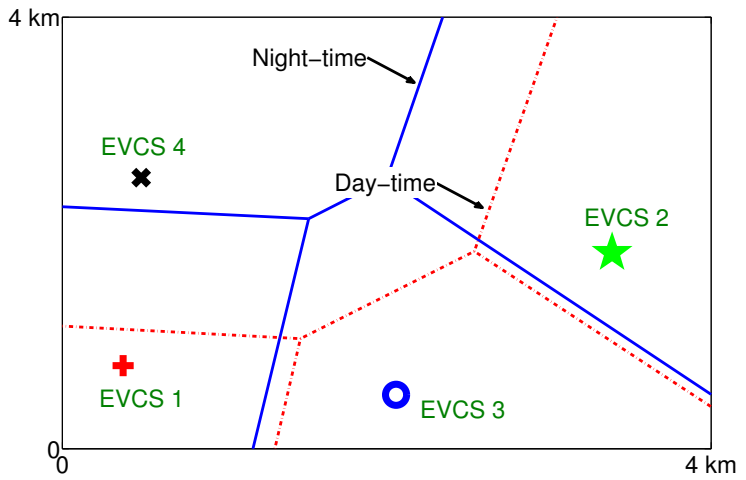

Fig. 2. Coverage of EVCSs.

generates $889 \mathrm{kWh}$ of electricity in 30 minutes during day time on average.

\section{B. Convergence of Price Adjustment Game}

First, we verify that the price adjustment game of EVCSs converges when best response algorithms are used. The position of the four EVCSs is depicted in Fig. 2. In this subsection, we assume that only EVCS 4 has a REGF, and EVCS 1, EVCS 2, and EVCS 3 do not have REGFs, to show the effect of EVCS with REGF on EVCSs without REGF.

In Fig. 2, the coverage areas of EVCSs during day time and night time are shown. In night time, EVCS 4 cannot generate electricity with the REGF. First, we can see that the boundaries of the coverage areas are straight lines, as we have shown in Lemma 1. Second, EVCS 4 has a larger coverage area in day time than in night time. In particular, the energy from the REGF leads to a lower electricity price, which helps to attract more EVs.

In Fig. 3, we show the electricity prices for EVCS 1 and EVCS 4 over the iterations of a best response algorithm. Herein, we consider the case where the REGF of EVCS 4 does not generate electricity initially but starts to generates electricity after 10 iterations (referred to as Case 1). We also consider the case where the REGF of EVCS 4 generates electricity from the beginning (referred to as Case 2). The initial electricity prices of the EVCSs are randomly chosen and the EVCSs update their electricity prices with probability of 0.7 every 30 minutes by using a best response algorithm.

We observe from Fig. 3 that the electricity prices of the EVCSs converge to the same values in both considered cases confirming the convergence of price adjustment game. We also observe that the electricity price of EVCS 4 with REGF is lower than that of EVCS 1 without REGF. Although not included in Fig. 3, the electricity prices of EVCS 2 and EVCS 3 are also higher than that of EVCS 4 . EVCS 4 reduces its electricity price to attract more EVs such that it can sell its excess electricity. Moreover, we can observe that the electricity price of EVCS 1 is affected by the price adjustment of EVCS 4. For Case 1, EVCS 4 reduces its price after 10 iterations, and then EVCS 1 also has to reduce its price accordingly, in order to avoid losing all of its customers.

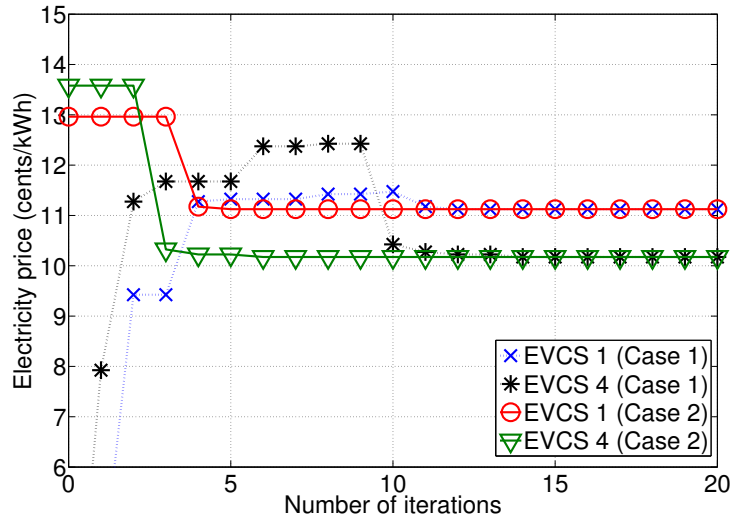

Fig. 3. Electricity prices vs. number of iterations.

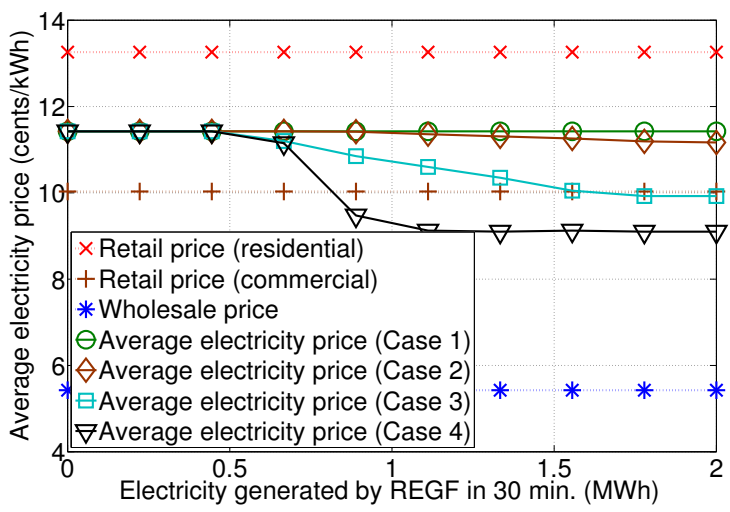

Fig. 4. Average electricity price of EVCS vs. electricity generated by REGF in 30 minutes.

\section{Effect of Renewable Power Generation at EVCS}

In Fig. 4, we show the average electricity price of EVCS 1 as a function of the electricity generated by the REGF in 30 minutes. Herein, we consider four cases: 1) none of the EVCSs has a REGF (referred to as Case 1), 2) EVCS 1 does not have a REGF but one of the other EVCSs has a REGF (referred to as Case 2), 3) only EVCS 1 has a REGF (referred to as Case 3), 4) all EVCSs have a REGF (referred to as Case 4).

We can observe from Fig. 4 that the average electricity price offered by EVCS 1 is always lower than the retail price of electricity for the residential sector. Therefore, EV owners can charge their vehicles at a lower rate by using an EVCS and this price difference justifies the usage of the EVCS. We can also observe that the average electricity price of EVCS 1 with REGF (i.e., Case 3 and Case 4) is lower than that of EVCS 1 without REGF (i.e., Case 1 and Case 2) when the generation is sufficiently large. Therefore, having renewable power generation with high power generation capacity is beneficial to the EVs, because the EVs can be charged at a lower price. Moreover, we can see that the average price of EVCS 1 with REGF decreases if all the other EVCSs have a REGF, because the competition between the EVCSs becomes more severe.

We also observe from Fig. 4 that the electricity price of EVCS 1 with REGF can be lower than the retail price for 


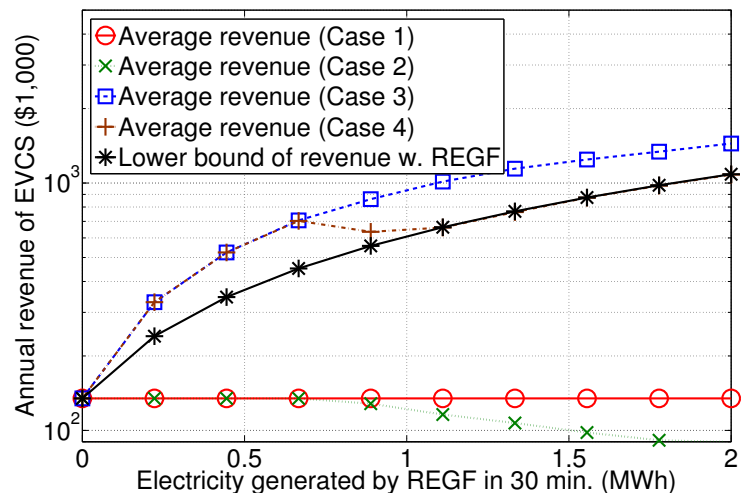

Fig. 5. Average annual revenue of EVCS vs. electricity generated by REGF in 30 minutes.

the commercial sector, which is the price for EVCSs to buy extra electricity from the power grid. However, in this case, EVCS 1 can still generate revenue because it can sell its excess electricity to EVs at a higher price compared to when it sells its electricity to the power grid.

In Fig. 5, we show the average annual revenue of EVCS 1 as a function of the electricity generated by its REGF in 30 minutes. The average annual revenue is calculated by taking into account the fact that a REGF cannot generate electricity during night time, such that the revenue during day time and night time is averaged. The lower bound in Fig. 5 corresponds to the revenue of EVCS 1 with a REGF when it sells all its excess electricity to the power grid and not to EVs. From Fig. 5 , we can see that the revenue of EVCS 1 is much higher if it has a REGF than if it does not, because if EVCS 1 has a REGF, it can sell its excess electricity to either the EVs or the power grid. EVCS 1 can generate more revenue by selling its electricity to EVs instead of the power grid. For example, assuming a REGF which generates $889 \mathrm{kWh}$ per 30 minutes, the revenue of the EVCS can be increased by $15 \%$, which corresponds to $\$ 78,000$ per year, if the EVCS sells its electricity to the EVs. In this case, the payback period ${ }^{4}$ of investing on REGFs is less than 2 years.

We observe that the revenue of EVCS 1 without REGF drops severely due to the price competition with the other EVCSs, which have a REGF. Therefore, if one EVCS introduces REGF, then the neighboring EVCSs are likely to add REGFs as well in order to avoid losing revenue. Therefore, eventually, all EVCSs will have their own REGF. Moreover, in Fig. 5, we can observe that the revenue of EVCS 1 for Case 4 converges to the lower bound when the electricity generated by the REGF is larger than 1.1 MWh due to the severe price competition between EVCSs.

\section{CONClusion}

In this paper, we explored the competition between EVCSs with renewable power generation in smart grid. We formulated the competitive price adjustment of EVCSs as a supermodular game. Based on the characteristics of supermodular games,

\footnotetext{
${ }^{4}$ The payback period is the time needed for the cumulative revenue to exceed the initial investment.
}

we showed that a unique pure Nash equilibrium exists for any best response algorithm with arbitrary initial policy. Through simulations, we confirmed the convergence of the price adjustment game. We also investigated the electricity price and the revenue of EVCSs as a function of the electricity generated from the renewable power generators and showed that adopting renewable power generation at the EVCSs can reduce the electricity price while increasing the revenue of the EVCSs. Thus, having renewable power generation at the EVCSs is beneficial for both the EVs and the EVCSs.

\section{REFERENCES}

[1] X. Fang, S. Misra, G. Xue, and D. Yang, "Smart grid the new and improved power grid: a survey," IEEE Communications Surveys \& Tutorials, vol. 14, no. 4, pp. 944-980, Oct. 2012.

[2] E. Sortomme, and M.A. El-Sharkawi, "Optimal charging strategies for unidirectional vehicle-to-grid," IEEE Trans. on Smart Grid, vol. 2, no. 1, pp. 131-138, Mar. 2011.

[3] A.J. Conejo, J.M. Morales, and L. Baringo, "Real-time demand response model," IEEE Trans. on Smart Grid, vol. 1, no. 3, pp. 236-242, Dec. 2010.

[4] R. Couillet, S.M. Perlaza, H. Tembine, and M. Debbah, "Electrical vehicles in the smart grid: A mean field game analysis," IEEE Journal on Selected Areas in Communications, vol. 30, no. 6, pp. 1086-1096, Jul. 2012.

[5] J. $\mathrm{Zu}$, and V.W.S. Wong, "An approximate dynamic programming approach for coordinated charging control at vehicle-to-grid aggregator," in Proc. of IEEE SmartGridComm, pp. 279-284, Oct. 2011.

[6] F. Soares, H. Morais, T. Sousa, Z. Vale, and P. Faria, "Day-ahead resource scheduling including demand response for electric vehicles," IEEE Trans. on Smart Grid, vol. 4, no. 1, pp. 596-605, Mar. 2013.

[7] Z. Fan, "A distributed demand response algorithm and its application to PHEV charging in smart grids," IEEE Trans. on Smart Grid, vol. 3, no. 3, pp. 1280-1290, Sep. 2012.

[8] W. Ko, and T.K. Hahn, "Analysis of consumer preferences for electric vehicles," IEEE Trans. on Smart Grid, vol. 4, no. 1, pp. 437-442, Mar. 2013.

[9] http://en.wikipedia.org/wiki/Electric_vehicle

[10] http://en.wikipedia.org/wiki/Charging_station

[11] http://www.eia.gov/electricity/

[12] A. Hess, F. Malandrino, M.B. Reinhardt, C. Casetti, K.A. Hummel, and J.M. Barcel-Ordinas, "Optimal deployment of charging stations for electric vehicular networks," in Proc. of the First Workshop on Urban Networking, pp. 1-6, Dec. 2012.

[13] I.S. Bayram, G. Michailidis, M. Devetsikiotis, S. Bhattacharya, A. Chakrabortty, and F. Granelli, "Local energy storage sizing in plugin hybrid electric vehicle charging stations under blocking probability constraints," in Proc. of IEEE SmartGridComm, pp. 78-83, Oct. 2011.

[14] M. Lewis, "Price dispersion and competition with differentiated sellers," The Journal of Industrial Economics, vol. 56, no. 3, pp. 354-678, Sep. 2008.

[15] T. Drezner, "Locating a single new facility among existing, unequally attractive facilities," The Journal of Regional Science, vol. 34, no. 2, pp. 237-252, May. 1994.

[16] E. Altman, and Z. Altman, "S-modular games and power control in wireless networks," IEEE Trans. on Automatic Control, vol. 48, no. 5, pp. 839-842, May 2003.

[17] E. Hong, S. Yun, and D. Cho, "Decentralized power control scheme in femtocell networks: A game theoretic approach," in Proc. of IEEE PIMRC, pp. 415-419, Sep. 2009.

[18] R.D. Yates, "A framework for uplink power control in cellular radio systems," IEEE Journal on Selected Areas in Communications, vol. 13 no. 7, pp. 1341-1347, Sep. 1995.

[19] http://www.dmv.state.pa.us/

[20] S. Han, S. Han, and K. Sezaki, "Development of an optimal vehicle-togrid aggregator for frequency regulation," IEEE Trans. on Smart Grid, vol. 1, no. 1, pp. 65-72, Jun. 2010.

[21] http://maps.nrel.gov/

[22] http://www.solarelectricsupply.com/commercial-solar-systems

[23] http://www.idc.lnt.de/uploads/media/leesmartgridcomm13.pdf 